Quim. Nova, Vol. 36, No. 1, 10-15, 2013

\title{
AVALIAÇÃO DA POTENCIALIDADE DE MICROALGAS DULCÍCOLAS COMO FONTE DE MATÉRIA-PRIMA GRAXA PARA A PRODUÇÃO DE BIODIESEL
}

\author{
Rafael Silva Menezes, Maria Inês Gonçalves Leles, Aline Terra Soares, Pedro Ivo Brandão e Melo Franco e Nelson Roberto \\ Antoniosi Filho* \\ Instituto de Química, Universidade Federal de Goiás, Campus II - Samambaia, CP 131, 74001-970 Goiânia - GO, Brasil \\ Célia Leite Sant'Anna
}

Seção de Ficologia, Instituto de Botânica, Secretaria do Meio Ambiente do Estado de São Paulo, Av. Miguel Estéfano, 3687, 04301-012 São Paulo - SP, Brasil

\section{Armando Augusto Henriques Vieira}

Departamento de Botânica, Centro de Ciências Biológicas e da Saúde, Universidade Federal de São Carlos, Rodovia Washington Luís, km 235, 13565-905 São Carlos - SP, Brasil

Recebido em 8/12/11; aceito em 9/8/12; publicado na web em 28/11/12

\begin{abstract}
EVALUATION OF THE POTENTIALITY OF FRESHWATER MICROALGAE AS A SOURCE OF RAW MATERIAL FOR BIODIESEL PRODUCTION. In this work, the fatty acid quantity and composition of six freshwater microalgae and soybean grains was determined by direct transesterification and gas chromatography analysis. The results showed that all the freshwater microalgae species presented a higher quantity of fatty acid than soybean grain. Choricystis $s p$. (A) provides $115 \%$ more fatty acids per gram of biomass than soybean grain. With regard to the fatty acid composition, Choricystis $s p$. (A) showed an adequate proportion of saturated and unsaturated fatty acids, with lower quantity of polyunsaturated fatty acids and, akin to some marine microalgae, constitutes an alternative raw material for biodiesel production.
\end{abstract}

Keywords: microalgae; transesterification; biodiesel.

\section{INTRODUÇÃO}

Atualmente, o Brasil se destaca por gerar $45 \%$ de sua matriz energética a partir de fontes renováveis de energia, com destaque para a produção de biocombustíveis. Entre os biocombustíveis mais promissores destaca-se o biodiesel, o qual no mundo é produzido basicamente utilizando óleos como os de soja, canola e palma. ${ }^{1}$ No caso do Brasil, a produção de biodiesel a partir da soja representa, aproximadamente, 80 a $90 \%$ da totalidade da matéria-prima graxa empregada para tal finalidade. ${ }^{2}$

A soja, contudo, apresenta pouco teor de óleo no grão e baixa produtividade por hectare, além de ser uma commodity agrícola. Essas características indicam que o aumento da produção de biodiesel no mundo tendo como base esta oleaginosa pode acarretar sérios danos econômicos e ambientais, especialmente se sua produção em larga escala for acompanhada do aumento da área cultivada, com expansão da fronteira agrícola e da ocupação humana via novos desmatamentos, com consequente perda de biodiversidade. ${ }^{2}$

Nesse sentido, é essencial pesquisar fontes de matérias-primas alternativas para a produção de biodiesel e, nesse contexto, destaca-se o potencial das microalgas. Um dos mais importantes apelos para produção de biodiesel a partir de microalgas é que este tipo de cultivo não deslocará as tradicionais áreas de cultivo voltadas para a alimentação humana - uma das principais críticas à produção de biodiesel a partir de plantas oleaginosas. ${ }^{3}$

Assim como as oleaginosas, muitas microalgas são ricas em triacilglicerídeos, os quais podem ser convertidos em ésteres metílicos de ácidos graxos (FAME - fatty acids methyl esters) para produção de biodiesel. Adiciona-se ainda o fato de que estudos demonstram que a composição química de ácidos graxos presentes em algumas microalgas é similar às oleaginosas atualmente utilizadas para a

*e-mail: nelson@quimica.ufg.br produção de biodiesel, tais como soja e canola. ${ }^{4,5}$

Atualmente o processo utilizado para a produção de biodiesel a partir de microalgas é a transesterificação do óleo extraído via catálise ácida ou básica na presença de um álcool. Na literatura são descritos vários processos de transformação de triacilglicerídeos em biocombustíveis, ${ }^{6}$ entretanto muitos são inviáveis economicamente devido ao alto custo da matéria-prima graxa. A fim de reduzir o custo da produção de biodiesel de microalgas e aumentar o rendimento em FAME, para essa biomassa se utiliza a transesterificação direta (TD), na qual a extração e transesterificação ocorrem simultaneamente numa única etapa. ${ }^{7,8}$

Apesar da abundância das microalgas dulcícolas, a maioria das microalgas utilizadas para a produção de biodiesel é de origem marinha. Um estudo com uma mistura de biodiesel contendo $20 \%$ de biodiesel feito a partir de Chlorella vulgaris em motores a diesel demonstrou a redução da emissão de dióxido de carbono e óxidos de nitrogênio em comparação ao diesel fóssil. ${ }^{9}$ Esta microalga é composta por $28,56 \%$ $(\mathrm{m} / \mathrm{m})$ de ácidos graxos saturados e $51,91 \%(\mathrm{~m} / \mathrm{m})$ de insaturados. ${ }^{10}$

O biodiesel produzido através da microalga Chlorella protothecoides apresentou propriedades de combustão similares ao diesel e aos parâmetros estabelecidos pela American Society for Testing and Materials (ATSM). ${ }^{11-13}$ Outra espécie que se tem mostrado como grande potencial para produção de biodiesel é a microalga Dunaliella tertiolecta.${ }^{14} \mathrm{~A}$ análise do perfil de ácidos graxos em $D$. tertiolecta mostrou teores de C18:3 e PUFA (polyunsaturated fatty acids) de aproximadamente 14,8 e $11,5 \%$, respectivamente. ${ }^{15}$ Apesar do elevado conteúdo em PUFA, esta microalga apresenta elevada taxa de crescimento e capacidade de crescimento em diferentes condições ambientais. ${ }^{14}$

Em relação à composição de ácidos graxos entre as diferentes espécies de microalgas marinhas, a maioria delas não atenderá os parâmetros exigidos por normas internacionais, devido apresentarem consideráveis teores de ácidos insaturados, especialmente tri- e poli-insaturados. Assim, diante das potencialidades de produção de biodiesel a partir de microalgas marinhas, estima-se que o biodiesel 
produzido por espécies dulcícolas demonstrará melhor qualidade nas propriedades físico-químicas do biodiesel. Além disso, deve-se levar em consideração que o perfil lipídico pode ser manipulado conforme as condições de cultivo, ${ }^{16}$ objetivando baixa produção de PUFA entre as espécies de interesse.

Considerando que o Brasil é um país de dimensões continentais e que algumas áreas de grande incidência de luz solar - essencial para a produção de microalgas - se encontram afastadas do oceano, é fundamental encontrar espécies de microalgas dulcícolas que possam ser produzidas nessas regiões situadas no interior do país.

Para que as microalgas dulcícolas se consolidem como uma alternativa viável é fundamental conhecer sua composição química a fim de que o biodiesel produzido alcance os padrões de qualidade especificados por normas internacionais, reguladas principalmente pela ASTM e European Standards (EN), como também pela Agência Nacional do Petróleo, Gás Natural e Biocombustíveis (ANP), no caso específico do Brasil. ${ }^{17}$

Assim, de acordo com os parâmetros estabelecidos busca-se selecionar espécies de microalgas que apresentem alta produtividade em ácidos graxos, os quais devem ser preferencialmente uma mistura de ácidos saturados e monoinsaturados, com reduzido teor de ácidos com duas ou mais duplas ligações, o que leva à obtenção de biodiesel com alta estabilidade oxidativa e adequada propriedade de congelamento.

Este trabalho teve como objetivo avaliar a adequabilidade de espécies de microalgas dulcícolas como matéria-prima graxa para a produção de biodiesel. Foram estudadas 6 taxa de microalgas dulcícolas oriundas da Coleção de Culturas de Microalgas de Água Doce da Universidade Federal de São Carlos (UFSCar). O potencial das espécies foi avaliado em relação ao rendimento e à composição de ésteres metílicos de ácidos graxos através da transesterificação direta da biomassa microalgal.

\section{PARTE EXPERIMENTAL}

\section{Microalgas dulcícolas}

Foram utilizadas as seguintes microalgas dulcícolas: Choricystis sp $A$ e $B$ (Chlorellaceae, Chlorococcales), isoladas do Rio Madeira (RO); Kirchneriella lunaris (Selenastraceae, Chlorococcales), isolada de um chafariz na cidade de São Carlos (SP); Kirchneriella irregularis (Selenastraceae, Chlorococcales), isolada de um lago de jardim na Escola Superior de Agricultura Luiz de Queiroz da Universidade de São Paulo (ESALQ-USP - Piracicaba, SP); Monoraphidium komarkovae (Selenastraceae, Chlorococcales), isolada do Pantanal Matogrossense (MT) e Tetranephris brasiliensis (Dictyosphaeriaceae, Chlorococcales), isolada da Represa de Guarapiranga (SP).

A identificação foi feita segundo critérios diacríticos descritos por Hindák. ${ }^{18-20}$

\section{Cultivos}

Após o isolamento com procedimentos mecânicos, ${ }^{21}$ as espécies foram cultivadas em frascos pirex de $2 \mathrm{~L}$ de capacidade com $1,8 \mathrm{~L}$ de meio WC, ${ }^{22}$ sob intensidade luminosa de aproximadamente 300 $\mu \mathrm{mol}$ fótons $\mathrm{m}^{-2} \mathrm{~s}^{-1}$, fornecida por tubos fluorescentes de $40 \mathrm{~W}$ em fotoperíodo de $12: 12 \mathrm{~h}$, e temperatura de $23 \pm 1{ }^{\circ} \mathrm{C}$. Os cultivos foram feitos com borbulhamento de ar enriquecido com $4 \%$ de $\mathrm{CO}_{2}$ a uma vazão de $0,1 \mathrm{~L} \mathrm{~min}^{-1}$.

No final da fase exponencial do crescimento, as células foram retiradas do meio de cultura através de filtração tangencial em cartucho de fibra oca. Posteriormente as amostras foram congeladas em nitrogênio líquido e, em seguida, submetidas à liofilização. Os volumes de cultivo usados para liofilização e as massas de microalgas

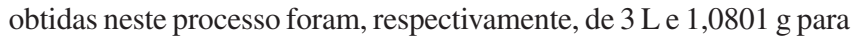
Choricystis (A); 3 L e 1,3337 g para Choricystis (B); 2 L e 0,8437 g para Kirchneriella lunaris; 3 L e 1,874 g para Kirchneriella irregularis; 2 L e 1,3698 g para Monoraphidium kormakovae e, 3 L e 1,3532 g para Tetranepris brasiliensis.

Após a liofilização, as amostras de microalgas foram acondicionadas em freezer.

\section{Determinação do teor de água por análise termogravimétrica}

As microalgas liofilizadas foram analisadas em analisador termogravimétrico da marca Mettler Toledo, modelo TGA/SDTA 851 ${ }^{\mathrm{e}}$, em cadinho de $\alpha$-alumina de $900 \mu \mathrm{L}$, com atmosfera de purga de

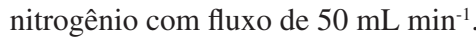

Os estudos termogravimétricos foram realizados de modo dinâmico, ${ }^{23}$ com determinação do teor de água utilizando a $1^{\mathrm{a}}$ derivada da curva TG para indicar os pontos de inicio e término do evento térmico, definido no intervalo de 30 a $100{ }^{\circ} \mathrm{C}$.

\section{Transesterificação direta (TD) da biomassa microalgal e de farelo de soja}

O rendimento e a análise do perfil de ésteres metílicos de ácidos graxos foram obtidos segundo método de Hartman e Lago adaptado para microescala. ${ }^{24}$

Inicialmente realizou-se o preparo de uma mistura esterificante, a qual foi utilizada no decorrer do processo de transesterificação. Para isso, adicionaram-se 2,0 g de cloreto de amônio (Merck ${ }^{\circledR}$ ) a $60 \mathrm{~mL}$ de metanol $\left(\right.$ Tedia $\left.^{\circledR}\right)$ seguido pela adição de 3,0 mL de ácido sulfúrico concentrado $\left(\right.$ Merck $\left.^{\circledR}\right)$. A mistura, contida em balão de fundo redondo adaptado a um condensador, foi mantida em refluxo sob agitação por 15 min. O reagente obtido foi então estocado em balão volumétrico de $100 \mathrm{~mL}$ com tampa de vidro.

Posteriormente, em um tudo de ensaio autoclavável de $20 \mathrm{~mL}$, pesou-se cerca de $200,0 \mathrm{mg}$ da biomassa de microalgas. A seguir, adicionaram-se $3,0 \mathrm{~mL}$ de solução $0,5 \mathrm{~mol} \mathrm{~L}^{-1}$ de hidróxido de sódio $\left(\right.$ Merck $\left.^{\circledR}\right)$ em metanol seco (Tedia ${ }^{\circledR}$ ) e aqueceu-se o tubo de ensaio por 10 min em banho-maria a $90^{\circ} \mathrm{C}$. Resfriou-se o tubo de ensaio em banho de gelo e adicionaram-se $9,0 \mathrm{~mL}$ da mistura esterificante previamente preparada segundo o procedimento descrito anteriormente. Aqueceu-se novamente o tubo de ensaio por $10 \mathrm{~min}$ em banho-maria a $90^{\circ} \mathrm{C}$. Resfriou-se o tubo de ensaio em banho de gelo e adicionaram-se 5,0 mL de n-heptano (Tedia ${ }^{\circledR}$ ) e 2,0 mL de água destilada. Agitou-se o tubo de ensaio algumas vezes e colocou-se o sistema em repouso até a separação de fases. A fase heptânica foi coletada com pipeta tipo Pasteur e analisada por cromatografia gasosa.

Para o cálculo do teor de éster da biomassa microalgal retirou-se $1 \mathrm{~mL}$ da fase heptânica, obtida via TD, e transferiu-se para frasco Eppendorf $^{\circledR}$ previamente pesado. As amostras foram deixadas em dessecador para evaporação do solvente até a massa de FAME atingir peso constante. O teor de éster de cada microalga foi obtido pela relação entre a massa de FAME no frasco Eppendorf ${ }^{\circledR} \mathrm{e}$ a massa inicial da biomassa utilizada no procedimento de TD. Neste cálculo subtraiu-se da massa inicial (cerca de $200 \mathrm{mg}$ ) a massa de água contida na microalga, a qual foi determinada por análise termogravimétrica (TG).

O procedimento de TD também foi adotado para a determinação do teor de ésteres em grãos de soja previamente triturados e secos em estufa.

\section{Análise cromatográfica dos FAME}

Para análise da composição de FAME e cálculo do teor de éster foi utilizado um cromatógrafo a gás Agilent 7890, equipado com detector 
FID e injetor split/splitless. A coluna capilar utilizada foi a DB-WAX (30 $\mathrm{m} \times 0,25 \mathrm{~mm} \times 0,25 \mu \mathrm{m}$ ). O forno operou sob temperatura inicial de $70{ }^{\circ} \mathrm{C}$, sendo aquecido a $10{ }^{\circ} \mathrm{C} \min ^{-1}$ até $240{ }^{\circ} \mathrm{C}$, e mantido nesta temperatura por $13 \mathrm{~min}$, sendo novamente aquecido a $5^{\circ} \mathrm{C} \mathrm{min}{ }^{-1}$ até $250^{\circ} \mathrm{C}$. O injetor foi mantido à temperatura de $310^{\circ} \mathrm{C}$, com volume de injeção de $2 \mu \mathrm{L}$, no modo split, com razão de split de 10:1. A temperatura do detector FID foi mantida a $310^{\circ} \mathrm{C}$. Hidrogênio 5,0 foi utilizado como gás de arraste à velocidade linear de $42 \mathrm{~cm} \mathrm{~s}^{-1} \mathrm{e}$ nitrogênio 5,0 foi usado como gás auxiliar a $20 \mathrm{~mL} \mathrm{~min}^{-1}$.

Os FAME foram identificados pela comparação direta com amostras de composição conhecida, tais como o óleo de soja, amendoim e crambre, pela análise de padrões de referência de FAME (Nu-Check Prep $^{\circledR}$ ) e por análises via cromatografia gasosa de alta resolução acoplada à espectrometria de massas (GC-MS), usando cromatógrafo a gás modelo Shimadzu 17A acoplado a espectrômetro de massas QP-5050 Shimadzu, com interface a $280{ }^{\circ} \mathrm{C}$. O gás de arraste utilizado nos ensaios foi o hélio 5,0 à velocidade linear de $42 \mathrm{~cm} \mathrm{~s}^{-1}$. As condições operacionais para forno, injetor e coluna capilar foram as mesmas utilizadas para HRGC-FID.

\section{RESULTADOS E DISCUSSÃO}

\section{Determinação do teor de água na biomassa microalgal}

A análise termogravimétrica das amostras de microalgas, exemplificada na Figura 1, demonstra que os processos de liofilização e armazenamento sob refrigeração fazem com que essas amostras apresentem baixos teores de água, os quais corresponderam a $1,78 \%$ em Kirchneriella lunaris, 2,36\% em Kirchneriella irregularis, 2,38\% em Monoraphidium komarkovae, 2,56\% em Tetranephris brasiliensis, 2,85\% em Choricystis sp. (A) e 3,93\% em Choricystis sp. (B). Esses valores foram utilizados na determinação da quantidade de biomassa seca utilizada no cálculo do teor de éster em cada microalga.

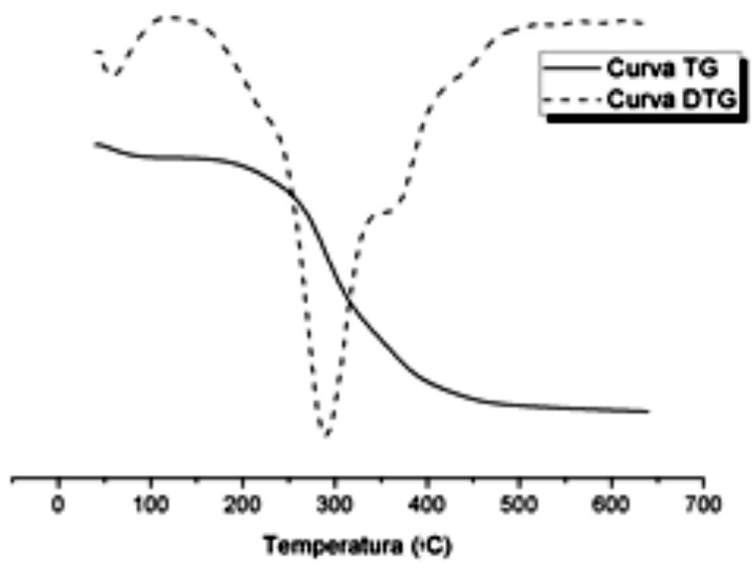

Figura 1. Análise termogravimétrica da biomassa da microalga dulcícola Chroricystis sp. (B)

\section{Composição de ácidos graxos}

A transesterificação direta da biomassa microalgal apresentou como vantagens a eliminação das etapas de extração e purificação do óleo, ${ }^{25}$ reduzindo o custo de produção e o tempo de reação. Além disso, todos os ácidos graxos - mesmo aqueles contidos em compostos minoritários com extremidade polar (como os lipídios ligados às proteínas), que normalmente não compõem um extrato oleoso - são convertidos a ésteres de ácidos graxos, o que maximiza a quantidade de biodiesel obtida a partir da biomassa microalgal.
Vale observar que, para a produção de biodiesel, atualmente se busca atingir elevada produção de óleo a partir de microalgas e, desta forma, conforme já mencionado, taxa marinhos são os mais comumente estudados, pois apresentam teores de óleos que podem variar em média de 20 a 50\% (porcentagem de óleo por biomassa seca), como o que ocorre em espécies dos gêneros Dunaliella, Chlorella, Tetraselmis, Neochloris, dentre outros. ${ }^{26}$ Entretanto, os métodos aplicados de determinação da quantidade de óleo são bastante diversos e, devido ao uso de solventes apolares e à alta quantidade de pigmentos lipofílicos existentes nessas amostras, elevados teores de óleos nem sempre correspondem a elevados rendimentos em ésteres. Assim sendo, para avaliar a potencialidade para a produção de biodiesel torna-se necessário avaliar o rendimento em ésteres, o que é obtido pela transesterificação direta da biomassa microalgal.

Pela transesterificação direta da biomassa das espécies, Choricystis sp. (A) foi a microalga que proporcionou o maior rendimento em ésteres metílicos de ácidos graxos, correspondendo a 422,9 $\mathrm{mg}$ de ésteres por grama de biomassa seca, o que é $115 \%$ maior do que o proporcionado pela soja (196,9 $\mathrm{mg}$ de ésteres por grama de biomassa seca), a qual é a oleaginosa mais utilizada para produção de biodiesel no Brasil. Neste sentido, todas as microalgas estudadas (Choricystis sp. (A), Choricystis sp. (B), Monoraphidium komarkovae, Kirchneriella lunaris, Kirchneriella irregularis e Tetranephris brasiliensis) apresentaram teores de ésteres maiores que o obtido para a soja (Figura 2).

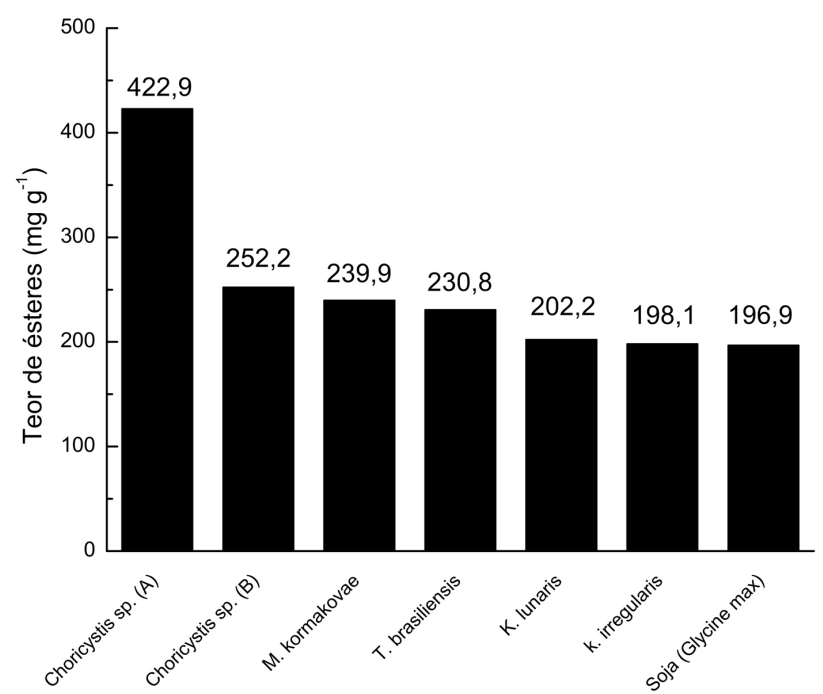

Figura 2. Rendimento em ésteres a partir da biomassa das microalgas dulcícolas

Ressalta-se que o rendimento em ésteres de Choricystis sp. (A) é aproximadamente $68 \%$ maior que o da microalga Choricystis $s p$. (B) que, apesar de ser de mesmo gênero, gerou 252,2 mg de ésteres por grama de biomassa seca.

De acordo com os dados da Tabela 1, os ácidos graxos comumente encontrados em todas as microalgas foram: mirístico (C14:0), palmítico (C16:0), palmitoleico (C16:1 cis9), hexadecenoico (C16:1 cis11), hexadecadienoico (C16:2 cis7, cis10); heptadecenoico (C:17:1 cis9), esteárico (C18:0), oleico (C18:1 cis9), vacênico (C18:1 cis11) e linoleico (C18:2 cis9, cis 12). Entre esses se destaca a presença em grande quantidade do ácido graxo palmítico que, nas microalgas estudadas, apresentou participação percentual variando de 24,7 a 35,6\% da composição total de FAME. Destaca-se ainda a variação percentual do teor de ácido oleico nas microalgas estudadas, para o qual o menor teor encontrado foi de $2,1 \%$ e o maior atingiu o patamar de $46 \%$. 
Tabela 1. Composição percentual dos ácidos graxos encontrados nas microalgas dulcícolas

\begin{tabular}{|c|c|c|c|c|c|c|}
\hline \multirow{2}{*}{ Ácidos graxos } & \multicolumn{6}{|c|}{ Composição para cada microalga (\%) } \\
\hline & $M k$ & $T b$ & $K i$ & $C A$ & $C B$ & $K l$ \\
\hline C6:0 & - & - & - & - & - & 0,7 \\
\hline $\mathrm{C} 8: 0$ & - & - & - & - & - & 0,9 \\
\hline C10:0 & - & - & - & 0,5 & 3,0 & 0,9 \\
\hline $\mathrm{C} 12: 0$ & - & - & - & 0,5 & 0,8 & 0,5 \\
\hline $\mathrm{M}(\mathrm{C} 14: 0)$ & 0,2 & 0,2 & 0,3 & 5,9 & 6,4 & 22,5 \\
\hline Mo (C14:1 cis 9$)$ & 0,2 & 0,2 & - & 3,1 & 2,6 & 1,1 \\
\hline $\mathrm{Pt}(\mathrm{C} 15: 0)$ & - & - & - & 1,5 & 1,6 & 1,1 \\
\hline $\mathrm{C} 15: 1$ cis 9 & 0,2 & - & - & 0,6 & 0,6 & 0,4 \\
\hline $\mathrm{P}(\mathrm{C} 16: 0)$ & 26,2 & 30,5 & 27,7 & 28,1 & 37,1 & 24,7 \\
\hline $\mathrm{C} 16: 1$ cis 7 & 0,2 & 0,3 & - & 2,4 & 0,5 & 2,3 \\
\hline Po $(\mathrm{C} 16: 1$ cis 9$)$ & 0,2 & 0,4 & 0,7 & 10,2 & 4,3 & 27,8 \\
\hline $\mathrm{C} 16: 1$ cis 11 & 0,2 & 0,3 & 0,3 & 0,6 & 0,7 & 2,8 \\
\hline $\mathrm{C} 16: 2$ cis 7, cis 10 & 0,9 & 0,6 & 1,2 & 1,3 & 0,8 & 1,1 \\
\hline $\mathrm{C} 16: 3$ cis 4, cis 7, cis 10 & - & - & 2,9 & - & 1,0 & - \\
\hline $\operatorname{Mg}(\mathrm{C} 17: 0)$ & 0,5 & - & 0,6 & 1,1 & 1,1 & 1,3 \\
\hline $\mathrm{C} 17: 1$ cis 9 & 1,0 & 1,3 & 0,9 & 2,4 & 0,7 & 2,0 \\
\hline $\mathrm{C} 17: 3$ cis 4, cis 7, cis 10 & 1,8 & 3,2 & - & - & - & 0,3 \\
\hline $\mathrm{S}(\mathrm{C} 18: 0)$ & 1,8 & 3,7 & 3,2 & 4,7 & 7,1 & 1,3 \\
\hline $\mathrm{C} 18: 1$ cis 7 & - & - & - & 1,0 & 2,4 & 1,2 \\
\hline $\mathrm{O}(\mathrm{C} 18: 1$ cis 9$)$ & 40,6 & 33,8 & 46,0 & 12,7 & 5,2 & 2,1 \\
\hline $\mathrm{V}(\mathrm{C} 18: 1$ cis 11$)$ & 0,4 & 0,5 & 0,3 & 5,1 & 2,5 & 1,6 \\
\hline $\mathrm{L}(\mathrm{C} 18: 2$ cis 9, cis 12$)$ & 7,2 & 11,0 & 2,8 & 6,8 & 1,6 & 1,8 \\
\hline$\gamma-\operatorname{Ln}(\mathrm{C} 18: 3$ cis6, cis9, cis 12$)$ & 0,2 & - & - & 0,5 & 0,3 & 0,3 \\
\hline $\operatorname{Ln}(\mathrm{C} 18: 3$ cis 9, cis 12 , cis 15$)$ & 12,7 & 12,4 & 10,4 & 3,4 & 1,9 & - \\
\hline $\mathrm{C} 18: 4$ cis 6, cis 9, cis 12, cis 15 & 3,8 & 1,6 & 2,7 & 0,4 & - & - \\
\hline C19:0 & - & - & - & 1,2 & 1,1 & - \\
\hline $\mathrm{C} 19: 1$ cis 9 & 1,4 & - & - & 0,9 & 3,2 & - \\
\hline C20:0 & - & - & - & 1,2 & 3,9 & - \\
\hline AA (C20:4 cis 5, cis 8, cis 11, cis 14$)$ & - & - & - & 1,0 & - & - \\
\hline EPA (C20:5 cis5, cis8, cis11, cis14, cis17) & 0,3 & - & - & 2,9 & 9,6 & 1,3 \\
\hline Saturados (SFA) & 28,7 & 34,4 & 31,8 & 44,7 & 62,1 & 53,9 \\
\hline Insaturados (UFA) & 71,3 & 65,6 & 68,2 & 55,3 & 37,9 & 46,1 \\
\hline Monoinsaturados (MUFA) & 44,4 & 36,8 & 48,2 & 39,0 & 22,7 & 41,3 \\
\hline Di-insaturados (DUFA) & 8,1 & 11,6 & 4,0 & 8,1 & 2,4 & 2,9 \\
\hline Tri-insaturados (TUFA) & 14,7 & 15,6 & 13,3 & 3,9 & 3,2 & 0,6 \\
\hline Poli-insaturados (PUFA) & 4,1 & 1,6 & 2,7 & 4,3 & 9,6 & 1,3 \\
\hline
\end{tabular}

(Mk): M. komarkovae; (Tb): T. brasiliensis; (Ki): K. irregularis; (CA): Choricystis sp. (A); (CB): Choricystis sp. (B); (Kl): K. lunaris.

A composição de ácidos graxos das microalgas Monoraphidium komarkovae, Tetranephris brasiliensis e Kirchneriella irregularis é caracterizada por elevados teores de ácidos como o palmítico, oleico e linolênico, seguidos pelos ácidos esteárico e linoleico em menores proporções. O perfil de ácidos graxos nestas espécies de microalgas é composto majoritariamente por ácidos graxos saturados $(28,7$ a $34,4 \%$ ) e monoinsaturados (34,4 a 36,8\%). Além disso, observou-se nestas amostras considerável presença de ácidos graxos com três ou mais duplas ligações. A presença considerável de ácidos graxos com ligações insaturadas também ocorre em microalgas marinhas. A microalga marinha Dunaliella tertiolecta - apontada como potencial matéria-prima graxa para a produção de biodiesel - apresentou teores médios de ácidos graxos saturados (SFA) de 28,8 e de 73,2\% para insaturados. ${ }^{14}$ As insaturações reduzem a viscosidade do biodiesel e melhoram as propriedades de ponto de entupimento de filtro a filtro. Entretanto, a presença de ácidos graxos insaturados, principalmente os PUFA, irá causar baixa estabilidade oxidativa ao biocombustível. ${ }^{27}$ Adicionalmente aos ácidos graxos mais comuns, foram encontrados nessas três espécies a presença de C18:4, como também C19:1 em M. komarkovae. A presença desses ácidos também foi identificada 
em espécies de microalgas dos gêneros Amphora, Chaetoceros, Chlorella, Dunaliella e Isochrysis. ${ }^{28}$

Em Choricystis sp. (A) $e(B)$ também se observou a presença de C19:1, como também de C19:0. A microalga Choricystis sp. (A) é majoritariamente composta pelos ácidos palmítico, palmitoleico e oleico (Figura 3). A razão entre o teor de ácidos graxos saturados $(44,7 \%)$ e monoinsaturados $(39,0 \%)$ é próxima de $1: 1$, além de apresentar baixo teor de TUFA $(3,9 \%)$ e PUFA $(4,3 \%)$. Isso é inferior aos teores de TUFA e PUFA obtidos para microalgas que apresentam potencial como fonte de matéria-prima para a produção de biodiesel, tais como Chlorella vulgaris (TUFA $=21,2 \%$ e PUFA $=4,8 \%$ ), Dunaliella tertiolecta $($ TUFA $=31,4 \%$ e PUFA $=10,6 \%$ ) e Neochloris oleabundans $(\mathrm{TUFA}=18,4 \%$ e PUFA $=9,3 \%) .{ }^{10}$

A predominância dos ácidos oleico e palmitoleico, dentre os ácidos insaturados de Choricystis sp. (A), e os baixos teores de di, tri e poli-insaturados favorecem algumas das propriedades físicas do biodiesel, tais como as de ponto de entupimento e viscosidade.

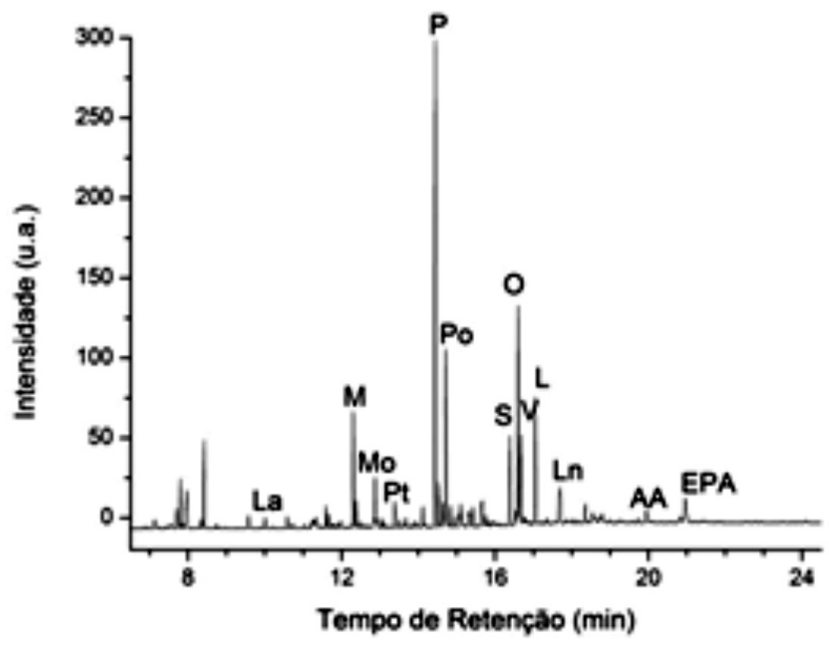

Figura 3. Cromatograma apresentando os principais ácidos graxos da microalga dulcícola Choricystis sp. (A)

A propriedade de índice de cetano, que mede a qualidade da combustão, está relacionada com a velocidade de ignição. Um alto índice de cetano indica bom funcionamento do motor, com minimização da quantidade de poluentes emitidos. Esta propriedade é influenciada pelo grau de saturação e comprimento da cadeia carbônica. Um biodiesel composto por ésteres de ácidos graxos saturados e de cadeia longa apresentará alto índice de cetano, o que favorece a qualidade do biocombustível. ${ }^{29}$ Portanto, os biodieseis das microalgas Kirchneriella lunaris e Choricystis sp. (A) e (B) apresentarão maiores índices de cetano comparativamente aos de $M$. komarkovae, $T$. brasiliensis e $K$. irregularis, por demonstrarem maior presença de ácidos saturados de cadeia média.

A microalga $K$. lunaris é caracterizada por ácidos graxos de cadeia curta a média, sendo que dentre esses últimos se destacam principalmente os ácidos mirístico, palmítico e palmitoleico. Observa-se que esta microalga é composta por baixos teores dos ácidos caproico $(0,7 \%)$ e caprílico $(0,9 \%)$, os quais não foram observados nas demais amostras. Desta forma, espera-se que o biodiesel da microalga $K$. lunaris apresente menor ponto de fulgor do que o das demais amostras, o que é prejudicial em termos de segurança, devido a maior inflamabilidade. Ainda com relação à microalga K. lunaris, o teor total de saturados em relação aos demais ácidos corresponde a 53,9\%, o que favorecerá a estabilidade oxidativa do biocombustível. Assim como em Choricystis sp. (A), em $K$. lunaris verificou-se uma razão entre teores de saturados e insaturados próxima de 1:1, com baixos teores de di-, tri- e poli-insaturados.

A microalga Choricystis sp. (B) apresentou perfil de ácidos graxos com maior percentual de ácidos graxos saturados em relação aos insaturados, correspondentes a um teor de 62,1 e 37,9\%, respectivamente. Diante dos efeitos discutidos anteriormente da presença de ácidos graxos saturados nas propriedades do biodiesel e considerando o elevado conteúdo de EPA $(9,6 \%)$, a aplicação desta microalga como fonte alternativa deste ácido essencial apresentará maior valor para as indústrias de fármacos e alimentos do que para a produção de biodiesel, pois o EPA é um agente importante para o sistema cardiovascular dos seres humanos. ${ }^{30}$

Avaliando os parâmetros estabelecidos pela norma EN 14214, o limite máximo estabelecido para o teor de ácido linolênico ou, no caso de microalgas, de ácidos tri-insaturados, é de $12 \%$; e de $1 \%$ para o teor máximo de ácidos graxos com mais de três duplas ligações (PUFA). Levando em consideração estas determinações, as microalgas $M$. komarkovae e $T$. brasiliensis não estão em concordância com o teor máximo de TUFA. Em relação à quantidade de PUFA, todas as microalgas apresentaram teores acima de $1 \%$, com destaque para a microalga $K$. lunaris, que apresentou teor de $1,3 \%$, o qual é próximo ao máximo estabelecido pela EN 14214 e similar ao encontrado em espécies marinhas do gênero Chlorella, ${ }^{28}$ cuja espécie Chlorella protothecoids propiciou a obtenção de biodiesel de qualidade adequada. ${ }^{13}$ Entretanto, no Brasil a normatização da ANP não estabelece limites máximos para teores de ácidos tri- e poli-insaturados. ${ }^{31}$

\section{CONCLUSÕES}

Levando em consideração a produção de biodiesel, o rendimento da conversão de lipídios em ésteres é de extrema importância para assegurar a rentabilidade do processo. Neste sentido, dentre as microalgas dulcícolas analisadas a Choricystis sp. (A) foi a que apresentou melhor rendimento na obtenção de ésteres metílicos de ácidos graxos, sendo este $115 \%$ superior ao apresentado pela soja. Ademais, todas as 6 espécies de microalgas estudadas forneceram teores de ésteres de ácidos graxos superiores ao fornecido pela soja via transesterificação direta da biomassa.

A microalga Choricystis sp. (A) também apresentou proporção adequada entre ácidos graxos saturados e monoinsaturados, e teores de ácidos graxos tri- e poli-insaturados inferiores a muitas microalgas marinhas apontadas como potenciais matérias-primas para a produção de biodiesel.

Desta forma, a microalga dulcícola Choricystis sp. (A), por apresentar elevados rendimentos em ésteres e adequada composição de ácidos graxos, demonstra potencialidade para ser utilizada como fonte de matéria-prima para a produção de biodiesel, o que é importante para a interiorização da produção desse biocombustível a partir de microalgas.

\section{AGRADECIMENTOS}

Ao Ministério da Ciência Tecnologia e Inovação (MCTI) ao apoio financeiro concedido por meio da FINEP (Convênio $n^{\circ}$ 01.10.0457.00) e do CNPq (Processo n ${ }^{\circ} 574796 / 2008-8$ ), à CAPES pela bolsa concedida a A. T. Soares, ao CNPq pela bolsa concedida para N. R. Antoniosi Filho (Produtividade em Pesquisa, Processo 309832/20101) e à FUNAPE pela administração dos recursos financeiros.

\section{REFERÊNCIAS}

1. Ferrari, R. A.; Oliveira, V. S.; Scabio, A.; Quim. Nova 2005, 28, 19.

2. Suarez, P. A. Z.; Santos, A. L. F.; Rodrigues, J. P.; Alves, M. B.; Quim. Nova 2009, 32, 768. 
3. Li, Q.; Du, W.; Liu, D.; Appl. Microbiol. Biotechnol. 2008, 80, 749.

4. Sheehan, J.; Dunahay, T.; Benemann, J.; Roessler, P.; NREL Report, 1998. (NREL/TP-580-24190).

5. Derner, R. B.; Ohse, S.; Villela, M.; Carvalho, S. M.; Fett, R.; Cienc. Rural 2006, 36, 1959.

6. Suarez, P. A. Z.; Meneghetti, S. M. P.; Meneghetti, M. R.; Wolf, C. R.; Quim. Nova 2007, 30, 667.

7. Armenta, R. E.; Scott, S. D.; Burja, A. M.; Radianingtyas, H.; Barrow, C. J.; Chromatographia 2009, 70, 629.

8. Ruiz, J. R.; Belarbi, E. H.; Sánchez, J. L. G.; Alonso, D. L.; Biotechnol. Technol. 1998, 12, 689.

9. Scragg, A. H.; Morrison, J.; Shales, S. W.; Enzyme Microb. Technol. 2003, 33, 884 .

10. Gouveia, L.; Oliveira, A. C.; J. Ind. Microbiol. Biotechnol. 2009, 36, 269.

11. Miao, X.; Wu, Q.; J. Biotechnol. 2004, 110, 85

12. Miao, X.; Wu, Q.; Bioresour. Technol. 2006, 97, 841.

13. Xu, H.; Miao, X.; Wu, Q.; J. Biotechnol. 2006, 126, 499.

14. Tang, H.; Abunasser, N.; Garcia, M. E. D.; Meng, C.; Simon, N. K. Y.; Salley, S. O.; Appl. Energy 2011, 88, 3324.

15. Campos, V. B.; Barbarino, E.; Lourenço, S. O.; Cienc. Rural 2010, 40 309.

16. Fidalgo, J. P.; Cid, A.; Torres, E.; Sukenik, A.; Herrero, C.; Aquaculture 1998, $166,105$.
17. Monteiro, M. R.; Ambrozin, A. R. P.; Lião, L. M.; Ferreira, A. G.; Talanta 2008, 77, 593.

18. Hindák, F.; Biol. Prace 1984, 1, 308

19. Hindák, F.; Biol. Prace 1988, 34, 263.

20. Hindák, F.; Biol. Prace 1990, 36, 225.

21. Vieira, A. A. H.; Bol. Inst. Oceanogr. 1977, 26, 303.

22. Guillard, R. R. L.; Lorenzen, C. J.; J. Phycol. 1972, 8, 10.

23. Faria, E. A.; Leles, M. I. G.; Ionashiro, M.; Zuppa, T. O.; Antoniosi Filho, N. R.; Eclet. Quím. 2002, 27, 111.

24. Antoniosi Filho, N. R.; Tese de Doutorado, Universidade Federal de São Carlos, Brasil, 1995.

25. Lewis, T.; Nichols, P. D.; Mcmeekin, T. A.; J. Microbiol. Methods 2000, $43,107$.

26. Chisti, Y.; Biotechnol. Adv. 2007, 25, 294.

27. Knothe, G.; Fuel Process. Technol. 2005, 86, 1059.

28. Knothe, G.; Green Chem. 2011, 13, 3048.

29. Knothe, G.; Matheaus, A. C.; Ryan, T. W.; Fuel 2003, 82, 971.

30. Ward O. P.; Singh, A.; Process Biochem. 2005, 40, 3627.

31. Agência Nacional do Petróleo, Gás Natural e Biocombustíveis - ANP; Resolução No 14, de 11/05/2012, Diário Oficial da União 18/05/2012 da. 